\title{
MICROLEAKAGE EVALUATION OF BULK-FILL COMPOSITE IN CLASS II RESTORATIONS OF PRIMARY MOLARS
}

\author{
Najla A. Eltoum ${ }^{1} B D S$, Niveen S. Bakry ${ }^{2} P h D$, Dalia M. Talaat ${ }^{3} P h D$, Sonia M. \\ Elshabrawy ${ }^{4} P h D$
}

\begin{abstract}
INTRODUCTION: Bulk-fill composites are promising restorative materials which may minimize placement time yet provide successful, durable esthetic restoration.

OBJECTIVES: The purpose of this in vitro study was to evaluate marginal microleakage of nano-hybrid bulk-fill composite, and compare it to incrementally placed conventional nano-hybrid composite when used to restore proximal cavities in primary molars.

MATERIALS AND METHODS: The sample consisted of 44 freshly extracted sound primary molars. Standardized proximal cavity preparations were prepared and the sample was divided randomly into 2 equal groups: Group I ( $\mathrm{n}=22)$ : Teeth were restored with nano-hybrid bulk-fill composite, Group II ( $\mathrm{n}=22)$ : Teeth were restored with incrementally placed conventional nano-hybrid composite. All specimens were thermocycled and immersed in $2 \%$ methylene blue dye at $37 \mathrm{C}^{\circ}$ for 24 hours. Methylene blue penetration was assessed on a 4 point grading scale (0 to 3) using a stereomicroscope to evaluate the microleakage of the restorations. Data were statistically analyzed using Mann-Whitney test and Wilcoxon Signed Ranks test.

RESULTS: No statistically significant difference has been found in the microleakage scores of group I at occlusal and cervical margin $(\mathrm{p}=1.000)$. In group II, no statistically significant difference has been found in the microleakage scores between occlusal and cervical margins $(\mathrm{P}=0.366)$. Comparing the microleakage scores of group I and group II, no statistically significant difference has been found ( $\mathrm{p}=0.240$ ). Although no significant difference was detected, bulk- fill composite showed lower microleakage scores than incrementally placed conventional composite.
\end{abstract}

CONCLUSIONS: Bulk-fill composite restoration marginal integrity was comparable to incrementally placed conventional composite in proximal cavities of primary molars.

KEYWORDS: composite, bulk-fill, microleakage, class II, primary molars.

1. B.D.S. Faculty of Dentistry, University of Science and Technology, Sudan.

2. Professor of Pediatric Dentistry, Faculty of Dentistry, Alexandria University, Alexandria, Egypt.

3. Associate Professor of Pediatric Dentistry, Faculty of Dentistry, Alexandria University, Alexandria, Egypt.

4. Professor of Dental Biomaterial, Faculty of Dentistry, Alexandria University, Alexandria, Egypt.

Corresponding author:

E-mail: najlaalzain@yahoo.com

\section{INTRODUCTION}

Restoration of carious primary teeth is significant not only for the healthy development and psychics of the child but also for the physiological development of permanent dentition. According to Poulsen et al, Pitts et al, and Guo et al in the years 2002, 2006 and 2013 respectively, a dramatic reduction in caries rates for permanent teeth has been reported in many industrialized countries in the past decades, but for primary teeth this fall has not occurred $(1,2)$.

Composite resins introduced by Bowen in 1962 (3), and become widely used dental materials since they restore both esthetics and function of dental tissues. They are expected to have physical properties comparable to those of tooth enamel and dentin and provide a long period of service (4). Despite its increasing use in the posterior teeth, these materials still present problems related to marginal integrity and leakage, mostly due to their inherent polymerization shrinkage (5).

The polymerization shrinkage can result in gap formation between composite resin and the cavity walls. Marginal gap formation contributes to microleakage permitting the passage of fluids, bacteria from the oral cavity and becomes a source of post-operative sensitivity, pulpal inflammation and recurrent caries (6).
In an attempt to decrease the effects of polymerization shrinkage, many clinical techniques have been proposed, such as incremental layering techniques introduced by Leader in 1948 (7), control of curing light irradiance introduced by Caulk in 1971 (7), flowable resin liner application, and increasing the filler content in its composition. However, no technique has been shown to be perfectly effective in reducing the effects of polymerization shrinkage (8).

In 2000's, a group of conventional composite resins called nano filled composites were introduced, which uses nanofiller technology and are formulated with nanocluster and nanomer filler particles. Nanoclusters are loosely bound agglomerates of nano-particles, and nanomers are discrete nano agglomerated particles of 20-75 $\mathrm{nm}$ in size (9). This formulation providing better physical properties and polish maintenance, and decreases the material deterioration over the years (10). However, the increment placement technique of composite resin may increase the possibility of voids formation between composite layers. Moreover, the risk of contamination leading to a compromised restoration is adversely impacted by the time it takes to place, adapt and cure each increment (11). As a result, many dentists impatiently anticipated the arrival of an alternative to this sensitive multiple layering technique (12). 
A new generation of so-called 'bulk-fill' composites has been introduced (13). These materials are suitable for insertion in a $4 \mathrm{~mm}$ bulk placement due to their reduced polymerization stress and their high reactivity to light curing (14). Consequently, problems arise from polymerization shrinkage and restoration time could be reduced without compromising the quality of cure $(4,13)$. They are used mainly in the posterior areas and considered to have superior physical and mechanical properties to combat higher masticatory forces (15). Moreover, the reduced treatment time decrease the risk of air entrapment or moisture contamination. They are also claimed to decrease the inward deformation of the cusps (cuspal deflection), and promote light transmittance (16).

Bulk-fill composites include flowable and high viscosity (packable) material types (4). Flowable bulk-fill composites are applied to basically replace dentin in one single layer of maximum $4 \mathrm{~mm}$; this bulk-fill base needs to be over-layered by a conventional composite to restore the tooth's outer anatomy, because this composite can exhibit low wear resistance (17). Packable bulk-full composites enable to restore the whole cavity in one single increment, with no need of an overlying final layer to complete anatomy (18).

Studies examining marginal adaptation and polymerization shrinkage with different materials and placement techniques have shown conflicting results. Some researchers reported that bulk-fill composites have lowered shrinkage stresses in comparison to a conventional composite (19-21), whereas other researchers found no statistically significant differences $(22,23)$. In terms of marginal adaptation, bulk-fill composites performed similar to incrementally placed conventional composite $(13,14$, 24).

The advanced development of bulk-fill composites needs continuous research and testing to approve these restorations and to overcome the problem of marginal microleakage. Therefore, there is a need to examine microleakage of bulk-fill composite in primary posterior teeth. The aim of this study was to evaluate the marginal sealing of nano-hybrid bulk-fill composite compared to incrementally placed conventional nano-hybrid composite in proximal restorations of primary molars.

The null hypothesis tested was no significant difference between the nano-hybrid bulk-fill composite and the incrementally placed conventional nano-hybrid composite regarding microleakage in proximal restorations of primary molars.

\section{MATERIALS AND METHODS}

This in vitro experimental study was performed in the Departments of Pediatric Dentistry and Dental Public Health and Dental Biomaterials, Alexandria University, after ethics committee approval.

The estimated sample size was 22 teeth per group using alpha error $=5 \%$, at a power $80 \%$, determined by $G$ Power software (25). The study sample included 44 non carious second primary molars extracted for orthodontic purpose or near their shedding time and stored in normal saline at room temperature. Teeth were free of cracks or enamel defects when examined by magnifying lens (8).

Standardized proximal cavities were prepared on either mesial or distal surface of each tooth using \#330 carbide bur. The bur was replaced after every four preparations. The occlusal part of proximal box was limited to the fossa triangularis. The width of the proximal box was extended from the central groove a distance equal to one bur diameter to the buccal and one bur diameter to the lingual. The proximal box depth was equal to $3 \mathrm{~mm}$ in height occlusocervically. The mesiodistal width of the gingival seat was approximately $1 \mathrm{~mm}$. For standardization purpose, a millimeter ruler and a K-fill were used. Teeth were then divided randomly into 2 equal groups (26).

Group I (n=22): Teeth were restored with Tetric EvoCeram bulk-fill composite (IvoclarVivadent, Schaan, Liechtenstein).

Group II (n=22): Teeth were restored with Grandio SO conventional composite (Voco, Guxhaven, Germany) (Table 1).

Table 1: Description of the different materials tested in this study

\begin{tabular}{|c|c|c|c|}
\hline Material & Manufacture & Type & Composition \\
\hline $\begin{array}{l}\text { Tetric Evo Ceram } \\
\text { (bulk-fill) }\end{array}$ & $\begin{array}{c}\text { IvoclarVivadent, } \\
\text { Schaan } \\
\text {,Liechtenstein }\end{array}$ & $\begin{array}{l}\text { Nano- } \\
\text { hybrid } \\
\text { composite }\end{array}$ & $\begin{array}{l}\text { Resin: dimethacrylates (Bis-GMA, } \\
\text { bis-EMA, UDMA) } \\
\text { Fillers: } 17 \% \text { prepolymers, Ba-Al-F } \\
\text { (particle size } 0.4-0.7 \mu \mathrm{m} \text { ), } \mathrm{YbF}_{3} \\
\text { (mean particle size } 200 \mathrm{~nm} \text { ) } \\
\text { Filler loading: } 61 \% \text { by weight }\end{array}$ \\
\hline ExciTE F & $\begin{array}{c}\text { IvoclarVivadent, } \\
\text { Schaan } \\
\text {,Liechtenstein }\end{array}$ & Adhesive & $\begin{array}{l}\text { phosphonic acid acrylate, HEMA, } \\
\text { dimethacrylate, highly dispersed } \\
\text { silicone dixoide, initiators, } \\
\text { stabilizers and potassium fluoride } \\
\text { in an alcohol solution. }\end{array}$ \\
\hline N-Etch & $\begin{array}{l}\text { IvoclarVivadent, } \\
\text { Schaan } \\
\text {,Liechtenstein }\end{array}$ & Etchant & $37 \%$ phosphoric acid \\
\hline $\begin{array}{c}\text { GrandioSO } \\
\text { (incremental-fill) }\end{array}$ & $\begin{array}{c}\text { Voco, } \\
\text { Guxhaven, } \\
\text { Germany }\end{array}$ & $\begin{array}{l}\text { Nano- } \\
\text { hybrid } \\
\text { composite }\end{array}$ & $\begin{array}{l}\text { Resin: Di and trimethacrylates (Bis- } \\
\text { GMA, TEGDMA,bis-EMA) } \\
\text { Fillers: Ba-Al-F(particle size } 1 \mu \mathrm{m} \text { ), } \\
\text { silicon dioxide (particle size of } 20- \\
40 \mathrm{~nm} \text { ) } \\
\text { Filler loading: } 89 \% \text { by weight }\end{array}$ \\
\hline Solobond M & $\begin{array}{l}\text { Voco, } \\
\text { Guxhaven, } \\
\text { Germany }\end{array}$ & Adhesive & $\begin{array}{l}\text { BIS-GMA, HEMA, phosphate } \\
\text { methacrylates, BHT, acetone, CQ, } \\
\text { amine accelerator }\end{array}$ \\
\hline Vococid & $\begin{array}{l}\text { Voco, } \\
\text { Guxhaven, } \\
\text { Germany }\end{array}$ & Etchant & $35 \%$ phosphoric acid \\
\hline
\end{tabular}

All prepared cavities were thoroughly cleaned with water and dried gently. Preparations were etched with phosphoric acid for 20 seconds, rinsed for 20 seconds, and blown dry with an air syringe. Total-etch adhesive were applied according to manufacturer's instructions and light cured from the occlusal direction on the proximal box. Etchants and adhesives were from the corresponding manufacturers of the composites to avoid possible incompatibilities (13). For group I: Bulk-Fill nano-hybrid composite (Tetric EvoCeram), was placed in one increment layer, then light cured from the occlusal direction for 20 seconds. For group II: Incrementally placed conventional nano-hybrid composite (GrandioSO), was placed in two horizontal increment layers. Each layer was light cured from the occlusal direction for 20 seconds (13).

All teeth were stored in distilled water for 24 hours at $37^{\circ} \mathrm{C}$ (27). Restored teeth were subjected to 1000 cycle between $5{ }^{\circ} \mathrm{C}$ and $55^{\circ} \mathrm{C}$ in controlled water bath with a dwell time of 30 seconds in each bath (28).

The entire surface of each tooth (with exception of the restorations and $1 \mathrm{~mm}$ around) were covered with double layer of nail polish to ensure a proof isolation of the teeth surfaces against dye penetration. All specimens were immersed in 2\% methylene blue solution for 24 hours at $37^{\circ} \mathrm{C}$. After dye exposure, the teeth were rinsed thoroughly 
under running water for half an hour, then bisected longitudinally in a mesisodistal direction parallel to the long axis through the center of the restoration with discs that were replaced every five specimens. The sectioning resulted in two approximately equal parts which were both analyzed for microleakage (27).

The cut surfaces of the sectioned teeth were examined and viewed under a stereomicroscope at a magnification of x20 to analyze the extent of dye penetration at occlusal and cervical margins of the restorations. Microleakage was assessed according to the scoring system described by Radhika et al in 2010 (29). The highest amount of microleakage was recorded as the scores of the restoration. The kappa statistic of intra-examiner reliability was 0.944 .

\section{Statistical analysis}

Statistical analysis was done using IBM SPSS software package version 20.0. (Armonk, NY: IBM Corp). Wilcoxon signed ranks test was used for abnormally distributed variables, to compare between occlusal and cervical margins. Mann Whitney test was used for abnormally distributed variables to compare between two study groups. The Kolmogorov-Smirnov test was used to verify the normality of distribution. Significance of the obtained results was judged at the $5 \%$ level (30).

\section{RESULTS}

Comparing the microleakage scores of group I at occlusal and cervical margin, no statistically significant difference has been found $(\mathrm{P}=1.000)$ (Figure 1). In addition, also in group II, no statistically significant difference has been found between occlusal and cervical margins $(\mathrm{P}=0.366)$ (Figure 2).

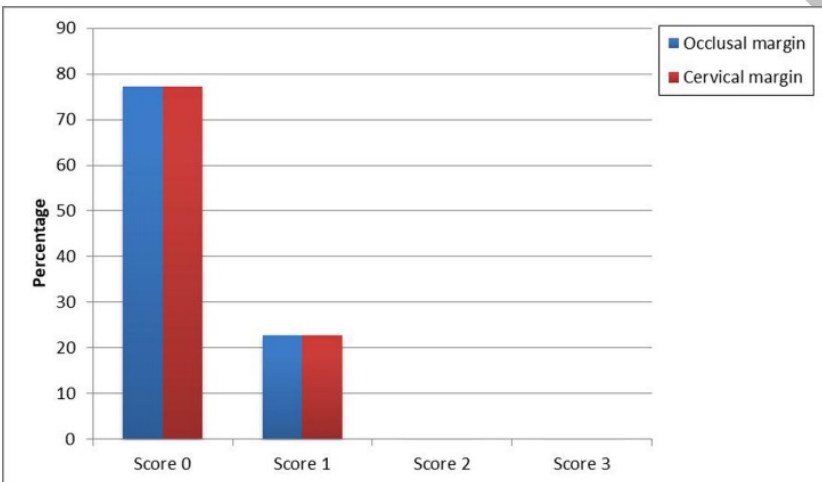

Figure 1: Comparison between occlusal margin and cervical margin according to microleakage score in group I (Tetric EvoCeram bulk-fill).

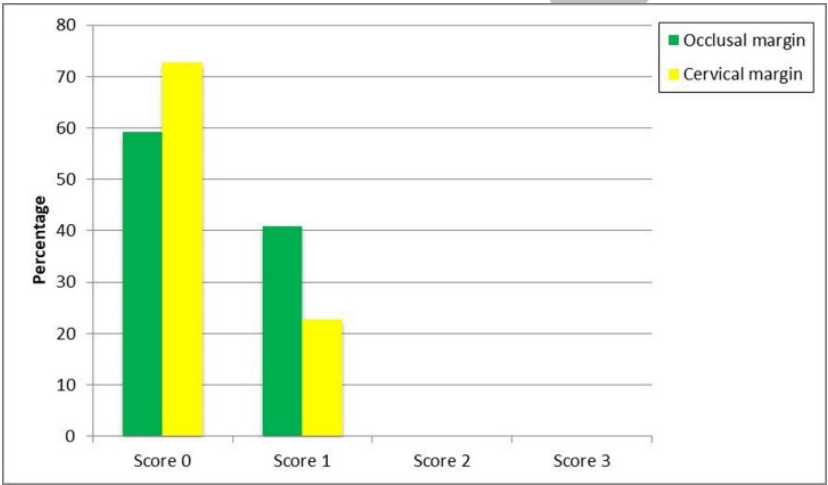

Figure 2: Comparison between occlusal margin and cervical margin according to microleakage score in group II (GrandioSO).
Comparing the combined occlusal and cervical microleakage scores of group I and group, bulk- fill composite showed lower microleakage scores than incrementally placed conventional composite with no statistically significant difference $(\mathrm{P}=0.240)$ (Table 2).

Table 2: Comparison between combined occlusal and cervical microleakage scores of group I and group II

\begin{tabular}{|c|c|c|c|c||}
\hline \multirow{2}{*}{ Microleakage score } & \multicolumn{2}{|c|}{$\begin{array}{c}\text { Bulk-fill } \\
\text { composite } \\
\text { (Group I) } \\
\text { (n= 44) }\end{array}$} & $\begin{array}{c}\text { Conventional } \\
\text { composite } \\
\text { (Group II) } \\
\text { (n= 44) }\end{array}$ \\
\cline { 2 - 6 } & No. & \% & No. & \% \\
\hline 0 & 34 & 77.3 & 29 & 65.9 \\
1 & 10 & 22.7 & 15 & 34.1 \\
2 & 0 & 0.0 & 0 & 0.0 \\
3 & 0 & 0.0 & 0 & 0.0 \\
\hline Min. - Max. & \multicolumn{2}{|c|}{$0.0-1.0$} & \multicolumn{2}{|c|}{$0.0-1.0$} \\
Median & \multicolumn{3}{|c|}{$0.058(0.240)$ NS } \\
\hline U (p) & \multicolumn{3}{|c|}{0.0} \\
\hline
\end{tabular}

$\mathrm{U}, \mathrm{p}$ : $\mathrm{U}$ and $\mathrm{p}$ values for Mann Whitney test for comparing between the two materials.

NS: Not statistically significant

\section{DISCUSSION}

The null hypothesis of the present study was supported by the results. Marginal integrity of bulk-fill composite did not differ from incrementally placed conventional composite in proximal restoration of primary molars.

Class II cavity preparation was considered the appropriate cavity preparation for testing the in vitro performance of composite resin, as the critical isthmus portion offers a challenging area for any restorative material. Additionally, the in accessibility of the cervical area in class II preparation and the problem of moisture control are additional factors that prove that class II preparation is the best design to evaluate marginal integrity of bulk- fill composite (31).

Aging restorations at body temperature and exposing them to thermocycling and/ or mechanical loading are treatment methods commonly used before in vitro microleakage testing to simulate intra-oral service life of a restoration (32).

Different regimens have been used for thermocycling of dental restorations with recommended temperatures ranging between $4^{\circ}$ and $60^{\circ} \mathrm{C}$. In the current study all specimens were subjected to 1000 cycles between $5^{\circ} \mathrm{C}$ and $55^{\circ} \mathrm{C}$ with dwell time 30 seconds which is considered an appropriate artificial aging test as it is equivalent to 12 months of clinical service (32).

The dye penetration method used in this study to measure sealing ability of the test composite restorations is the most popular, and mainly performed after cutting the teeth in a longitudinal direction. Since the size of oral bacteria is of magnitude larger than dye particle, the penetration of the dye can be regarded as an early indication of compromised margins. Various dyes can be used such as methylene blue, basic fuchsin, India ink, and silver nitrate with developer. Out of the various studies methylene blue has been proved to be useful aid. Methylene blue (2 \%) was 
used in this study because of its low cost, low molecular weight of the dye, and ease of application (33).

Regarding the effect of margin location, the results of the present study showed no significant difference between occlusal and cervical margins microleakage score in the bulk-fill composite group (test group), and incrementally placed conventional composite (control group). This finding was in agreement with Campos et al (14), who found no significant differences between the occlusal and cervical microleakage scores before/after thermomechanical loading when they used bulk-fill composite in class II permanent molars. Comparable results were obtained by Rad et al (34), who examined the microleakage of two bulk-fills and one conventional composite at two different time points (24 hours, 3 months) in class II cavities in premolar teeth. This finding might be due to the high and reliable dentin bond strength of the used adhesives. On the contrary Mosharrafian et al (35), found that microleakage scores in the cervical margins were significantly greater than that in the occlusal margins for both bulk-fills and incrementally placed conventional composite in class II restorations of primary posterior teeth. Variations in findings can be related to differences in materials, dye used, different bonding agents, and different thermocyclic protocols (24).

The present data showed no statistically significant difference between the combined occlusal and cervical microleakage scores of the tested groups. These results might be explained by the similar mechanical properties and consistency of the two materials (nanohybrid packable composites), and by the use of adhesive systems that involve acid etching of enamel and dentin. This finding is in agreement with the results obtained by Do et al (13), who evaluated the cuspal flexure, depth of cure, and microleakage of bulk-fill composites and incrementally placed conventional nanocomposite as control in permanent molars. This finding is also in agreement with Benetti et al (36), who compared the depth of cure, polymerization contraction, and microleakage of bulkfill composites with those of incrementally placed conventional composite in permanent molars. Comparable results were obtained by Heintz et al (37); they claimed that the marginal integrity of medium sized class II restorations of composite resins placed in bulk was similar to that of composite restorations placed incrementally in permanent molars.

Polymerization shrinkage is responsible for the formation of internal stresses in the material and leakage between the filling and the walls of the cavity. According to Campos et al (14), Van Ende et al (38), It has been hypothesized that the elastic modulus is more important than shrinkage in determining the stress, in which, the greater the elasticity of the material in the cavity, the greater the reduction in polymerization shrinkage stresses. This also may explain lack of a significant difference in microleakage of bulk-fill and incrementally placed conventional composites in our study, although the least microleakage score was in the bulk-fill composite group. According to the information provided by the manufacturers, modulus of elasticity and filler load of incrementally placed conventional composite ( $89 \%$ by weight) are greater than that of bulk-fill composite ( $61 \%$ by weight) used in the current study. It means that incrementally placed conventional composite has lower elasticity. When polymerized, there is a possibility that bulk-fill composite show higher elasticity than incrementally placed conventional composite and thus, reduction in polymerization shrinkage stresses occurs to a greater extent, preventing microleakage in use of bulk-fill composite (34).

On the contrary, a study carried out by Miletic et al (39), compared strain, displacement, as well as microleakage of bulk-fill (FiltekBulk Fill Posterior, Tetric EvoCeram Bulk Fill, fiber-reinforcedEverX Posterior, giomer Beautifil Bulk), lowshrinkage (Kalore, GC), and conventional nanohybrid (Tetric EvoCeram), microhybrid (Filtek Z250), in modified Class II cavities in permanent molars. They found that, incrementally placed conventional composites resulted in less gingival leakage than bulk-fill composites.

Bulk-fill composite may be useful in restoring primary molars especially when time is of importance while working with children. In addition, the cervical portion of proximal box most distant from light source will have better curing with bulk-fill composite. One limitation of this study that should be mentioned is the fact that the research is conducted in vitro. It is likely that these results predicted the performance of composite restorations but they cannot account for all the potential influences of the oral environment in vivo. As reported by Pashly in 1990 (40), the results of an in vitro microleakage study should be viewed as a theoretical maximum level of leakage more than that may be expected in vivo. Therefore, future research can contribute to better understanding of clinical performance, longevity, and efficacy of these bulk-fill restorations.

The null hypothesis of the present study was supported by the results. Marginal integrity of bulk-fill composite did not differ from incrementally placed conventional composite in proximal restoration of primary molars.

Class II cavity preparation was considered the appropriate cavity preparation for testing the in vitro performance of composite resin, as the critical isthmus portion offers a challenging area for any restorative material. Additionally, the in accessibility of the cervical area in class II preparation and the problem of moisture control are additional factors that prove that class II preparation is the best design to evaluate marginal integrity of bulk- fill composite (31).

Aging restorations at body temperature and exposing them to thermocycling and/ or mechanical loading are treatment methods commonly used before in vitro microleakage testing to simulate intra-oral service life of a restoration (32).

Different regimens have been used for thermocycling of dental restorations with recommended temperatures ranging between $4^{\circ}$ and $60^{\circ} \mathrm{C}$. In the current study all specimens were subjected to 1000 cycles between $5^{\circ} \mathrm{C}$ and $55^{\circ} \mathrm{C}$ with dwell time 30 seconds which is considered an appropriate artificial aging test as it is equivalent to 12 months of clinical service (32).

The dye penetration method used in this study to measure sealing ability of the test composite restorations is the most popular, and mainly performed after cutting the teeth in a longitudinal direction. Since the size of oral bacteria is of magnitude larger than dye particle, the penetration of the dye can be regarded as an early indication of compromised margins. Various dyes can be used such as methylene blue, basic fuchsin, India ink, and silver nitrate with developer. Out of the various studies methylene blue has been proved to be useful aid. Methylene blue (2\%) was 
used in this study because of its low cost, low molecular weight of the dye, and ease of application (33).

Regarding the effect of margin location, the results of the present study showed no significant difference between occlusal and cervical margins microleakage score in the bulk-fill composite group (test group), and incrementally placed conventional composite (control group). This finding was in agreement with Campos et al (14), who found no significant differences between the occlusal and cervical microleakage scores before/after thermomechanical loading when they used bulk-fill composite in class II permanent molars. Comparable results were obtained by Rad et al (34), who examined the microleakage of two bulk-fills and one conventional composite at two different time points (24 hours, 3 months) in class II cavities in premolar teeth. This finding might be due to the high and reliable dentin bond strength of the used adhesives. On the contrary Mosharrafian et al (35), found that microleakage scores in the cervical margins were significantly greater than that in the occlusal margins for both bulk-fills and incrementally placed conventional composite in class II restorations of primary posterior teeth. Variations in findings can be related to differences in materials, dye used, different bonding agents, and different thermocyclic protocols (24).

The present data showed no statistically significant difference between the combined occlusal and cervical microleakage scores of the tested groups. These results might be explained by the similar mechanical properties and consistency of the two materials (nanohybrid packable composites), and by the use of adhesive systems that involve acid etching of enamel and dentin. This finding is in agreement with the results obtained by Do et al (13), who evaluated the cuspal flexure, depth of cure, and microleakage of bulk-fill composites and incrementally placed conventional nanocomposite as control in permanent molars. This finding is also in agreement with Benetti et al (36), who compared the depth of cure, polymerization contraction, and microleakage of bulkfill composites with those of incrementally placed conventional composite in permanent molars. Comparable results were obtained by Heintz et al (37); they claimed that the marginal integrity of medium sized class II restorations of composite resins placed in bulk was similar to that of composite restorations placed incrementally in permanent molars.

Polymerization shrinkage is responsible for the formation of internal stresses in the material and leakage between the filling and the walls of the cavity. According to Campos et al (14), Van Ende et al (38), It has been hypothesized that the elastic modulus is more important than shrinkage in determining the stress, in which, the greater the elasticity of the material in the cavity, the greater the reduction in polymerization shrinkage stresses. This also may explain lack of a significant difference in microleakage of bulk-fill and incrementally placed conventional composites in our study, although the least microleakage score was in the bulk-fill composite group. According to the information provided by the manufacturers, modulus of elasticity and filler load of incrementally placed conventional composite ( $89 \%$ by weight) are greater than that of bulk-fill composite ( $61 \%$ by weight) used in the current study. It means that incrementally placed conventional composite has lower elasticity. When polymerized, there is a possibility that bulk-fill composite show higher elasticity than incrementally placed conventional composite and thus, reduction in polymerization shrinkage stresses occurs to a greater extent, preventing microleakage in use of bulk-fill composite (34).

On the contrary, a study carried out by Miletic et al (39), compared strain, displacement, as well as microleakage of bulk-fill (FiltekBulk Fill Posterior, Tetric EvoCeram Bulk Fill, fiber-reinforcedEverX Posterior, giomer Beautifil Bulk), lowshrinkage (Kalore, GC), and conventional nanohybrid (Tetric EvoCeram), microhybrid (Filtek Z250), in modified Class II cavities in permanent molars. They found that, incrementally placed conventional composites resulted in less gingival leakage than bulk-fill composites.

Bulk-fill composite may be useful in restoring primary molars especially when time is of importance while working with children. In addition, the cervical portion of proximal box most distant from light source will have better curing with bulk-fill composite. One limitation of this study that should be mentioned is the fact that the research is conducted in vitro. It is likely that these results predicted the performance of composite restorations but they cannot account for all the potential influences of the oral environment in vivo. As reported by Pashly in 1990 (40), the results of an in vitro microleakage study should be viewed as a theoretical maximum level of leakage more than that may be expected in vivo. Therefore, future research can contribute to better understanding of clinical performance, longevity, and efficacy of these bulk-fill restorations.

\section{CONCLUSION}

Within the limitations of the present study, it is possible to conclude that bulk-fill composite restoration marginal integrity was comparable to incrementally placed conventional composite in proximal cavities of primary molars.

\section{CONFLICT OF INTEREST}

The authors declare that they have no conflicts of interest.

\section{REFERENCES}

1. Yoonis E, Kukletová M. Tooth-colored dental restorative materials in primary dentition. Scripta Medica. 2009; 82:108-114.

2. Chadwick BL, Evans DJ. Restoration of class II cavities in primary molar teeth with conventional and resin modified glass ionomer cements: a systematic review of the literature. Eur Arch Paediatr Dent. 2007; 8:14-21.

3. Bowen RL. Composite and sealant resins-past, present, and future. Pediatr Dent. 1982; 4:10-5.

4. Abed YA, Sabry HA, Alrobeigy NA. Degree of conversion and surface hardness of bulk-fill composite versus incremental-fill composite. Tanta Dent J. 2015; 12:71-80.

5. de Andrade OS, Mario F, Montes MA. Marginal adaptation and microtensile bond strength of composite indirect restorations bonded to dentin treated with adhesive and lowviscosity composite. Dent Mater. 2007; 23:279-87.

6. Poggio C, Chiesa M, Scribante A, Mekler J, Colombo M. Microleakage in Class II composite restorations with margins below the CEJ: In vitro evaluation of different restorative techniques. Med Oral Patol Oral Cir Bucal. 2013; 18:793-8. 
7. Aschheim, Kenneth W. Esthetic dentistry: A clinical approach to techniques and materials. 3rd ed., St. Louis, Elsevier Mosby, 2015.

8. Webber, Marin, Progiante, Marson. Bulk-fill resin-based composites: Microleakage of class II restorations. JSCD. 2014; 2:15-9.

9. Jain A, Deepti D, Tavane PN, Singh A, Gupta P, Gupta A, Sonkusre S. Evaluation of microleakage of recent nanohybrid composites in class V restorations: An in vitro study. Int J Adv Health Sci. 2015; 2:8-12.

10. Gonçalves MA, Teixeira VC, Rodrigues SS, de Oliveira RS, Salvio LA. Evaluation of the roughness of composite resins submitted to different surface treatments. Acta Odontol Latinoam. 2012; 25:89-95.

11. Opdam NJ, Roeters JJ, Peters TC, Burgersdijk RC, Teunis M. Cavity wall adaptation and voids in adhesive Class I restorations. Dent Mater. 1996; 12:230-5.

12. Lowe RA. Advances in composite resin materials. The material science behind modern restoratives. INSIDE DENTISTRY. 2015; 11:1-6.

13. Do T, Church B, Veríssimo C, Hackmyer SP, Tantbirojn D, Simon JF, Versluis A. Cuspal flexure, depth-of-cure, and bond integrity of bulk-fill composites. Pediatr Dent 2014; 36:468-73.

14. Campos EA, Ardu S, Lefever D, Jassé FF, Bortolotto T, Krejci I. Marginal adaptation of class II cavities restored with bulk-fill composites. J Dent. 2014; 42:575-81.

15. Al Azmi MM, Hashem MI, Assery MK, Al Sayed MSE. An in-vitro Evaluation of the mechanical properties and surface roughness of bulk fill vs incremental fill resin composites. Int J Prev Clin Dent Res. 2017; 4:1-6.

16. Abouelleil H, Pradelle N, Villat C, Attik N, Colon P, Grosgogeat B. Comparison of mechanical properties of a new fiber reinforced composite and bulk filling composites. Restor Dent Endod. 2015; 40:262-70.

17. Bunek SS. Update on Composites. The Dental Advisor. 2015; 32:2-7.

18. Colak H, Tokay U, Uzgur R, Hamidi MM, Ercan E. A prospective, randomized, double-blind clinical trial of one nano-hybrid and one high-viscosity bulk-fill composite restorative systems in class II cavities: 12 months results. Niger J Clin Pract. 2017; 20:822-31.

19. El-Damanhoury H, Platt J. Polymerization shrinkage stress kinetics and related properties of bulk-fill resin composites. Oper Dent. 2014; 39:374-82.

20. Kim H, Park S. Measurement of the internal adaptation of resin composites using Micro-CT and Its correlation with polymerization shrinkage. Oper Dent. 2014; 39:57-70.

21. Fronza BM, Rueggeberg FA, Braga RR, Mogilevych B, Soares LES, Martin AA. Monomer conversion, microhardness, internal marginal adaptation, and shrinkage stress of bulk-fill resin composites. Dent Mater. 2015; 31:1542-51.

22. Tiba A, Zeller GG, Estrich CG, Hong A. A Laboratory evaluation of bulk-fill versus traditional multi-incrementfill resin-based composites. J Am Dent Assoc. 2013; 144:1182-3.

23. Kim RJ, Kim Y, Choi N, Lee I. Polymerization shrinkage, modulus, and shrinkage stress related to tooth-restoration interfacial debonding in bulk-fill composites. J Dent. 2015; 43:430-9.

24. Al-Harbi F, Kaisarly D, Bader D, Gezawi ME. Marginal integrity of bulk versus incremental fill class II composite restorations. Oper Dent. 2016; 41:146-56.
25. Daniel W. Biostatistics. A foundation for analysis in health science $7^{\text {th }}$ ed. New York, Chichester, Brisbane: John Wiley and Sons.Inc.1999.

26. El-Negoly SA, Ibrahim FM, Ellatif AA. Immunohistochemical expression of tooth pulp dendritic cells as a response to ceramic reinforced glass ionomer restorative materials. E.D.J. 2009; 55: 93-102.

27. Miwan S. Abdul-Rahman. Evaluation of microleakage in the gingival margin of class II resin composite restoration when using three placement techniques. (An in vitro study). Sulaimani Dent J. 2014; 1:21-28.

28. Muangmingsuk A, Senawongse P, Yudhasaraprasithi S. Influence of different soft start polymerization techniques on marginal adaptation of class V restorations. Am J Dent 2003; 16:117-9.

29. Radhika M, Sajjan GS, Kumaraswamy BN, Mittal N. Effect of different placement techniques on marginal microleakage of deep class II cavities restored with two composite resin formulations. J Conserv Dent. 2010; 13: 915.

30. Kirkpatrick LA, Feeney BC. A simple guide to IBM SPSS statistics for version 20.0. Student ed. Belmont, Calif.: Wadsworth, Cengage Learning; 2013.

31. Hunt PR. A modified class II cavity preparation for glass ionomer restorative materials. Quintessence Int Dent Dig. 1984; 15: 1011-8.

32. Wahab FK, Shaini FJ, Morgano SM. The effect of thermocycling on microleakage of several commercially available composite Class V restorations in vitro. J Prosthet Dent. 2003; 90:168-74.

33. Patel MU, Punia SK, Bhat S, Singh G, Bhargava R, Goyal $\mathrm{P}$, et al. An in vitro evaluation of microleakage of posterior teeth restored with amalgam, composite and zirconomer A Stereomicroscopic Study. J Clin Diagn Res. 2015; 9:657.

34. Kianvash Rad N, Javid B, Panahandeh N, Ghasemi A, Kamali A, Mohammadi G. Microleakage of bulk-fill composites at two different time points. J Dent Sch 2016; 34: 225-34.

35. Mosharrafian S, Heidari A, Rahbar P. Microleakage of two bulk fill and one conventional composite in class II restorations of primary posterior teeth. J Dent (Tehran). 2017; 14:123-31.

36. Benetti A, Havndrup-Pedersen C, Honoré D, Pedersen M, Pallesen U. Bulk-Fill Resin Composites: Polymerization Contraction, Depth of Cure, and Gap Formation. Oper Dent. 2015; 40:190-200.

37. Heintze SD, Monreal D, Peschke A. Marginal quality of class II composite restorations placed in bulk compared to an incremental technique: Evaluation with SEM and stereomicroscope. J Adhes Dent. 2015; 17:147-54.

38. Van Ende A, De Munck J, Lise DP, Van Meerbeek B. Bulkfill composites: A review of the current literature. J Adhes Dent. 2017; 19:95-109.

39. Miletic V, Peric D, Milosevic M, Manojlovic D, Mitrovic N. Local deformation fields and marginal integrity of sculptable bulk-fill, low-shrinkage and conventional composites. Dent Mater. 2016; 32:1441-51.

40. Pashley DH. Clinical consideration of microleakage. J Endodont. 1990; 16:70-7. 